Pneumologe 2016 $\cdot 13: 293$

DOI 10.1007/s10405-016-0059-9

Online publiziert: 27. Juni 2016

(c) Springer-Verlag Berlin Heidelberg 2016

CrossMark

\author{
J. Lorenz' $\cdot$ F. J. Meyer ${ }^{2}$ \\ 'Klinik für Pneumologie, Internistische Intensivmedizin, Infektiologie und Schlafmedizin, Klinikum \\ Lüdenscheid, Lüdenscheid, Deutschland \\ ${ }^{2}$ Klinik für Pneumologie, Gastroenterologie, Internistische Intensiv- und Beatmungsmedizin, Klinikum \\ Harlaching und Klinik für Pneumologie und Pneumologische Onkologie, Klinikum Bogenhausen, \\ Lungenzentrum München, München, Deutschland
}

\title{
Pneumologische Intensivmedizin
}

Trotz erheblicher Fortschritte in der Betreuung kritisch kranker Patienten bestehen in der Intensivmedizin immer noch zahlreiche bekannte und auch neue Herausforderungen. Besondere Aufmerksamkeit verlangen Patienten mit vorgeschädigten Lungen, die eine maschinelle Atemhilfe benötigen und Patienten mit schwerer Sepsis oder septischem Schock, oftmals aufgrund einer komplexen Infektion mit multiresistentem Erreger.

Patienten mit Lungenfibrosen als Grunderkrankung zeichnen sich dadurch aus, dass profibrotische Signaltransduktionswege bereits aktiviert sind. Jeder neue unphysiologische mechanische Stimulus führt mit hoher Wahrscheinlichkeit zu einer Exazerbation mit rascher Entwicklung eines Lungenversagens. An diesem Problem haben auch lungenprotektive Verfahren der maschinellen Ventilation nichts geändert. Wegen der besonderen Bedeutung dieser Frage in der pneumologischen Intensivmedizin haben wir zwei Beiträge dieser Frage gewidmet.

Die Autoren J. Wälscher und Mitarbeiter aus Heidelberg greifen die Intensivtherapie von Patienten mit interstitiellen Lungenerkrankungen in allen ihren Facetten auf. Besondere Aufmerksamkeit verdient die Differenzialdiagnose der akuten Verschlechterung, denn neben akuten Exazerbationen der Grunderkrankung sind Rechtsherzdekompensationen bei pulmonaler Hypertonie, Lungenarterienembolie, Medikamenten und Verschlechterung von Komorbiditäten $\mathrm{zu}$ berücksichtigen. Der Einsatz der maschinellen Beatmung ist zurückhaltend $\mathrm{zu}$ erwägen und Alternativen sind zu bedenken.
Im Zusammenhang mit möglichen Alternativoptionen bei respiratorischem Typ-I-Versagen beschäftigen sich J. H. Storre und W. Windisch aus Köln mit einer neuen, aus der Pädiatrie kommenden und in diesem Setting bedeutenden Option, der „High-Flow“Sauerstofftherapie. Neben dem hohen Sauerstofffluss können weitere Eigenschaften des Verfahrens wie die Minimierung des physiologischen Totraumes und die Schaffung eines positiven endexspiratorischen Druckes genutzt werden. Einzelheiten der Methode und die inzwischen vorliegende Evidenz in der Erwachsenenintensivmedizin werden in dem Beitrag eingehend dargestellt.

\section{》) Bei pneumologischen Intensivpatienten kann eine Exazerbation schnell zum Lungenversagen führen}

Fast zwei Drittel aller Fälle mit schwerer Sepsis bzw. mit septischem Schock sind die Folge einer Pneumonie, in der Regel im ambulanten Umfeld erworben. M. Kolditz, Mitverfasser der kürzlich erschienen S3-Leitlinie zur ambulant erworbenen Pneumonie, berichtet über die aktuellen Standards zum Management dieser vital bedrohlichen Infektion. Ein Schwerpunkt im klinischen Alltag ist die möglichst frühe Identifikation betroffener Patienten durch Scores bzw. Bündel einfach und rasch zu erhebender Kriterien, die inzwischen in ihrer Vorhersagekraft gut evaluiert sind. In der Betreuung sind die etablierten Sepsisbündel anwendbar, während in der Therapie das Erregerspektrum der schweren am- bulant erworbenen Therapie berücksichtigt werden muss. Eingehend wird der Einsatzadjuvanter Therapien, wie dervon Kortikosteroiden, diskutiert und auf die Bedeutung und therapeutische Berücksichtigung kardiovaskulärer Komplikationen eingegangen.

In dem Beitrag von $H$. von Baum und $H$. Bracht über die klinische Infektiologie auf der Intensivstation steht das Konzept des „Antibiotic Stewardship“ im Vordergrund. Es zielt darauf, mittels medizinischer sowie prozeduraler Standards und mit Hilfe eines trainierten Mitarbeiterteams die Qualität der Verordnung von Antiinfektiva zu verbessern. Qualitätsindikatoren sind dabei Therapieergebnisse, Resistenzentwicklung und der Verbrauch von Antiinfektiva. Dies wird anhand des gelebten Modells im Universitätsklinikum Ulm praxisnah dargestellt.

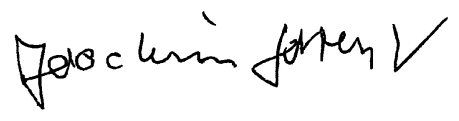

Prof. Dr. Joachim Lorenz

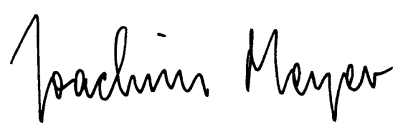

Prof. Dr. F. Joachim Meyer

\section{Korrespondenzadresse}

\section{Prof. Dr. J. Lorenz}

Klinik für Pneumologie, Internistische Intensivmedizin, Infektiologie und Schlafmedizin, Klinikum Lüdenscheid Paulmannshöherstraße 14,58515 Lüdenscheid, Deutschland

J.S.Lorenz@t-online.de

Interessenkonflikt. J. Lorenz und F.J. Meyer geben an, dass kein Interessenkonflikt besteht. 